\title{
How Blockchain-based Solutions Impact the Healthcare Sector
}

Anna Laesser*

The Startup Digest section introduces startups and grassroots initiatives from around the world that push the boundaries of emerging technologies. Most conversations around emerging technologies are stuck in silos and are quite hyped, making it hard to understand their actual impact on businesses, society and governance. The Startup Digests aim to demystify what is happening on the ground by establishing a discourse via case studies and interviews with startups and grassroots initiatives. Each edition will take a critical look on how these movements apply emerging technologies to achieve a specific purpose - facilitating a discourse that makes the (new) thinking, the approach and potential impact become more tangible.

This edition takes a closer look at how blockchain-based solutions affect the healthcare industry. Three case studies have been selected to exemplify how blockchain can potentially transform healthcare supply chain, patient data management, and access to health care. Each case study will be critically analysed to identify the potentials, challenges and risks.

\section{Introduction}

The healthcare industry is transforming. New and more efficient healthcare record systems are being developed, artificial intelligence and big data is being utilised to improve medical examinations and predict disease, wearable devices are integrated to track a patient's health and discussions around highly controversial genetic engineering are being held. ${ }^{1}$ However, the digitalization of healthcare faces a common challenge: moving data between multiple stakeholders within a health supply chain. The incorruptible distributed ledger technology, known as Blockchain, could offer a way to redesign the information architecture and potentially enable data integrity, transparency and security - as well as new business models. ${ }^{2}$ According to a study commissioned by IBM, around $16 \%$ of healthcare executives are determined to implement blockchain solutions this year, while around $56 \%$ expected to adopt blockchain by the year 2020. ${ }^{3}$

This Startup Digest consists of three case studies that will reveal the diversity of blockchain-based approaches in healthcare and how they are being used to rethink data access, data ownership and analytics. To reflect the buzz in this novel field, the selected projects are in different levels of development, namely a blockchain technology company (1), a research project supported by the MIT Media Lab (2), and an early stage startup (3). The following section will analyse the context, potential and risks of each blockchain-based solution and draw some conclusions.

The three case studies are:

1) Guardtime Health: A blockchain system for a connected healthcare supply chain (Estonia, Netherlands, United Kingdom; blockchain technology company)

DOI: 10.21552/delphi/2019/1/11

* Anna Laesser, Co-founder at Impact Hub Berlin. For correspondence: <anna.laesser@impacthub.berlin>

1 David Roberts, '10 ways digital could transform healthcare' (2018) <https://www.ey.com/en_gl/digital/10-ways-digital-could -transform-healthcare> accessed 1 March 2019.

2 Lisk Academy, 'Blockchain in Healthcare' (2018) <https://lisk.io/ academy/blockchain-basics/use-cases/blockchain-healthcare > accessed 1 March 2019.

3 Constantin Yukhymenko, 'What are the use cases for blockchain tech in healthcare?' (2018) < https://www.quora.com/What-are -the-use-cases-for-blockchain-tech-in-healthcare/answer/ Constantin-Yukhymenko>> accessed 1 March 2019. 
2) MedBloc: A 'Swiss Bank' for patient's medical records (USA, Massachusetts; MIT Media Lab research project)

3) Hanai: An exchange network for women to access healthcare information in rural areas via blockchain (Berlin, San Francisco; early stage startup)

\section{Interview I: Guardtime - Blockchain for a Connected Healthcare Supply Chain}

Name of your startup: Guardtime Health Location: Estonia, Netherlands, United Kingdom Your name, position: Glen Ogden, General Manager

Question: What is the challenge in healthcare that Guardtime Health is solving?

Guardtime has solved the data access challenges preventing the healthcare industry moving to much needed solutions such as Value Based Contracting, Complex Clinical Trials and Longitudinal Patient Records.

Question: What is your solution to efficiently manage supply-chains? What differentiates Guardtime from others? What differentiates Guardtime from others?

Guardtime extends existing supply chain technology, providing data integrity and immutability of every action, from first to last mile, without the need for all stakeholders to share or have access to a common supply chain platform. Guardtime's differentiator aside from the platform is we have been in production since 2012 with both a Global and Federal presence.

Question: Why/how did you decide to combine healthcare and blockchain technology? What are currently your main opportunities and challenges?

Guardtime's KSI Blockchain went into production with the Estonian government, securing the majority of the countries public databases, in 2012. In 2016, we went live securing the integrity of health data for their entire population, over 1.3m patient records. Guardtime's KSI Blockchain hashes every record and links every update to a given record providing complete end to end integrity with immutable provenance. Records are continually verified ensuring the veracity of patient data at all times with complete transparency of data usage. Our current focus is rolling out this solution with key industry partners to provide outcomes based contracting / value based agreements and complex clinical trial solutions with the necessary blockchain backed data access infrastructure.

Question: How does your solution disrupt the healthcare industry and patient engagement? How do you set yourself apart from similar approaches?

We disrupt healthcare not only through the functionality and scalability of the platform we've built but through key partnerships with industry leaders which enable us to bring together the right healthcare stakeholders. Regardless of the approach, Guardtime has been in production for many years at both a sovereign and enterprise level. This ability to showcase real-world scalable production case studies really sets us apart.

Question: How does your solution disrupt the healthcare industry and patient engagement? How do you set yourself apart from similar approaches?

The main challenge was finding the right partner who could provide the necessary level of access to the NHS primary care systems needed to make the solution work. The benefits of this solution are broad, from enabling patients to have a blockchain backed longitudinal record of their own health record which is updated daily that they can carry with them at all times to ensuring they're fully aware of their care pathway for a given condition with automatic diarised appointments based on NICE (National Institute of Care Excellence) standard's. General practitioners can be alerted when their patients miss appointments and take action if needed to ensure patients have the best level of care.

Question: How do you build trust and encourage patients to follow a personalized treatment plan? What is the benefit for patients?

On boarding patients to the system happens via their General Practitioner. Their GP encourages them to join as it's a very simple yet comprehensive way of managing their condition. The automation built in- 
to the system means patients don't have to worry about their care pathway as this is provided for them with the required appointments diarised.

Question: How do you ensure real-time data for clinical trials that are relevant for stakeholders (e.g. health care providers, insurers, and life science companies) and at the same time comply with health data processing?

The platform brings together all relevant stakeholders enabling both data access at rest and sharing of data if appropriate. To meet data protection regulations in certain locations a dynamic consent engine can be enabled ensuring patients consent to and know where their data is going at all times. The opportunities for this are huge as dynamic data access, at point of need backed with blockchain integrity and consent ensures data is actionable by all parties. We are very fortunate to have a range of industry leading partners who help us overcome the main challenge for these solutions which is bringing together the right stakeholders.

Question: Do you foresee any challenges between blockchain and GDPR in the future?

For DLT solutions there could be a challenge, especially around the right to be forgotten. Guardtime's blockchain doesn't suffer these challenges and this has allowed us to build a specific product for GDPR called Volta which ensures enterprise compliance with GDPR.

Question: How do you incorporate wearables and mobile devices to enhance patient reported outcomes that are relevant for accurate clinical trials?

The platform can be configured to take data from any source; however for clinical trials it is usual for a specific app/ wearable to be nominated. We have a range of APIs and SDKs which enable rapid on boarding of new technologies or apps for patient reported outcomes so a trial owner can choose either off the shelf or bespoke mechanisms to capture data. The critical component here is being able to guarantee the data came from the expected source and is verifiable. Moreover, it is crucial that the integrity of the data is immutable preventing tampering of PRO data after the fact.
Question: How do you track drugs in real-time between patient and pharma to prevent counterfeit drugs? What are the advantages/disadvantages of blockchain?

Guardtime's solutions are extensible and augment supply chain solutions to enable pharma to link serialised medications at source to the blockchain to prevent illegitimate over production whilst ensuring downstream stakeholders like pharmacists or patients can scan medication to check it's a genuine product. The primary advantages are this doesn't disrupt the existing supply chain technology making it easy to deploy and configure. As the platform allows for decentralised participation it removes the traditional problems faced by blockchain as it's typically not possible to force all stakeholders to use a single centralised platform.

Question: How does Guardtime Health impact existing and new business models in healthcare?

Data access is the number one problem everyone talks about in this space. We believe we've solved this problem in a decentralised way and by adding data protection regulation compliance and dynamic consent for patients we can both extend existing business models or support brand new novel models as necessary.

Question: What motivates you to push the boundaries of the health industry? What are your aspirations / future plans?

Our motivation is twofold. First, that data should be seen as a new form of medicine. Currently data sits in many silos and it's too voluminous to be pushed to a central data lake even if privacy regulations allowed it. If we can bring this data together without the need for a central repository where patients' privacy isn't compromised think how many new cures or treatments we will enable this industry to find. Secondly, there is nothing more personal to an individual than their health and patients need to have greater control over their health data whether this is to inform healthier behaviour or to help treat an existing condition. If we can bring this data together for a patient and give them a longitudinal view of their health records it means they're better informed and if they do need treatment should aid in faster diagnosis. Our 
future plans are to continue working with our partners to ensure a fully joined up eco-system bringing together all stakeholders in the patient care pathway, whether that's enabling patients to access the latest medication via outcome based pricing, or enabling complex clinical trials.

\section{Interview II: MedRec - A 'Swiss Bank' for Patient's Medical Records}

Name of your startup: MedRec

Location: Massachusetts, USA

Your name, position: Andy Lipman, Principal Investigator; MIT Media Lab

Question: What is the challenge in healthcare that MedRec aims to solve?

Increasingly, people are becoming responsible for their own healthcare. Simultaneously, their records are becoming available to them. Therefore, the challenge is to provide a simple, convenient way for people to determine who can access these diverse records wherever they are.

Question: What is your solution to improve health records and medical research data? What is unique about your approach?

One solution is to build a 'Swiss Bank' where you can deposit records and authorize their transmission. That risks adding an intermediary, or a set of them. This may not solve the problem. We elected to build a decentralized interchange system instead that does not require all healthcare providers to use a common data access control system. It is distributed and owned by all.

Question: Why/how did you decide to combine healthcare and blockchain technology?

We constructed a private blockchain that contains 'smart contracts'. These contracts define who can access specific records and under what conditions. The ledger of contracts is the blockchain and that in turn is maintained by providers. The blockchain is regulated by 'proof of authority' and is thereby efficient and robust enough for the application. Most of the providers are trusted entities (you already trust them with your data...), so we protect against intrusion and bad actions by a minority. The only cost to providers is to maintain the blockchain, which is not large, and to program an interface to their database that obeys the rules of the smart contracts.

Question: How does your prototype potentially disrupt the current healthcare industry and patient engagement?

I would hope it doesn't disrupt healthcare at all. The whole idea is to be easy to adopt and adapt to. The potential disruption and the most difficult part is the patient interface. Patients have to accept their responsibility to be in charge of their data. As with retirement plans, most people don't think about this until it is an issue.

Question: How do you aim to overcome the challenge of interoperability between different institutions such as providers, hospital-, and healthcare systems to share health data effectively?

MedRec does not contain medical data, only permissions. There is no need to replicate the data as long as we can access it as needed. Other organizations are working on interoperability of the data and common ways to describe it. We are not boiling that ocean.

Question: How do you aim to connect patients and stakeholders (e.g. health care providers, insurers, life science companies etc) to prioritize patient agency?

Initially, this is not an issue. Currently, data is transferred either by the patient themselves (transporting a fax or a CD), or by a blanket authorization given at the time of treatment. MedRec automates this and also allows unanticipated requests, like a ski accident.We have not considered other aspects of health such as insurers and life sciences companies, although that can be potentially included. Insurers can participate and be authorized by contract. We envision that patients might also want to participate in clinical trials or statistical analyses. We have not debugged that, but an easy way would be to requests to be routed through legitimate providers.

Question: Looking at the EU, it would be very useful for patients to be able to bring their health records with them when they live in another member state. 
How would MedRec approach this? How would you deal with GDPR?

We do not hold records. No reason why contracts could not adhere to common laws and norms.

Question: How does MedRec potentially impact existing and new business models in healthcare?

The hard part of this kind of work is getting it started. One way might be to ally with major records producers now. In the US, there are Cerner and EPIC for example. MedRec can be a consumer front end for them.

Question: What motivates you to push the boundaries of the health industry? What are your aspirations / future plans for MedRec?

We think this is an interesting area for exploring the use of a distributed system. One thing we learned was that the blockchain can act as an agent for interoperability: In the case of Medrec, the provider interface module is the only element added; there need be no other changes to internal operations. That module, by translating contracts into database requests is in effect a compatibility layer between diverse data bases.

\section{Interview III: Hanai - A Healthcare Information Network for Rural Areas via Blockchain}

Name of your startup: Hanai

Location: Silicon Valley to London, Luxembourg, Berlin, Porto

Your name, position: Shamala Hinrichsen, CEO

Question: What challenge in healthcare is Hanai aiming to solve?

No child should die of malnutrition and no mother should die of pregnancy related-complications. Especially if it is due to pure lack of information and/or access to healthcare. Traversing rural farming communities across the vast sub-continent of India, spanning languages, climates, diets, people, the people stood out in their one reason to live - to make the lives of their children better. In that one grand de- sire, often people take great risks, get into debt, sacrifice too much of themselves. What if there was a way to, at the very least, empower them with a way of knowing what is required for their health, their children's health, their community's health. What if this can be done simply, through their mobile phones. What if this can be a way to get them to participate in healthcare efforts, use hospitals, not rely on themselves, but a system. Be it a millet farmer in Jawhar, India, or a vegetable seller in Chibuto, Mozambique, the need is similar, and the need is basic - health and better health.

Question: What are the key insights and aha-moments that you gained during your fieldwork that made you choose a blockchain-based solution?

On my first field visit to India, my second son was six-months old and I was still breastfeeding. So often, I would bump into women who had babes hanging from their breasts as Nils was as well. In that one picture frame, we were all the same. Our worries, concerns, moments of joy - the same. But were we the same? Not on many fronts. I have access and the ability to access information on what is best to feed my six month old, when it is an emergency, what are the vaccinations he needs. The more I listened, the more it hit me, that while entire government-driven initiatives, multiple NGOs and global healthcare organisations continue to engage with rural communities, no one knows the measure of their impact. Not much work was done to capture data. And not much was being done to just look simply at how a woman accesses media, technology and how to leverage that. For the end user, our client, who is usually from a side-lined proportion of the population, but yet with access to mobile technology-, it does not matter how the tech behind works, or that it is even tech. And there was the other thing that made me and every other breastfeeding mother I met similar - we all used smart phones.

\section{Question: What is your solution?}

Hanai is a blockchain backed, cloud-based fully transparent marketplace of tools to deliver, measure and improve upon social impact, especially in healthcare. Through decentralised identities we empower individuals to control their own data while giving organ- 
isations clarity on their impact. In simpler terms, we provide a dashboard, the tech for any organisation to deliver healthcare information, measure the data, improve upon their work, engage hospitals, get a noncash based payment system going, and involve token economics.

Question: Can you elaborate on the three technical elements that Hanai is exploring? For example, blockchain, artificial intelligence and cloud-based technology.

a. Blockchain: People often think we are jumping on the tech bandwagon with what is currently sexy. The reality is we cannot continue to work with marginalized communities and see to their healthcare needs in the same way we have been. We have to be bold. And blockchain which offers immutability and transparency, gives us a way to deal with sensitive information like patient data. It is also gives us an opportunity to explore healthcare funding while cutting out corruption.

b. Artificial intelligence: We are in an era of Digital Exponential. I think it is no longer the time to look at what cannot be digital or can never be but ride the wave-responsibly, critically, and in a civil manner. It needs to be a good balance of human emotional intelligence working in parallel with AI. As we already know now, many decisions have to be led by AI - like hiring practises etc. But at the same time, it cannot be only AI. With healthcare, we need to approach cautiously, but we can already use AI to experiment besides just predict where disease outbreaks are happening. This is such a simple example of many.

c. Cloud-based system: We knew no other way, within our backgrounds, brainstorming. We continue to explore and learn. Team Hanai is constantly engaged in conversation and we are open to people approaching us to completely knock us off our chairs.

Question: How do you bring together all three technologies? What are your key learnings in integrating them? What is most critical to be successful?

The tech is there to be stretched and used to solve issues. That is the way Hanai sees it, so a few years down the line, we may have dropped something and picked up something new. To be successful: we learn, learn, learn and focus on the people, the people, the people. If you know you are learning to help people, your solution will iterate, flex and mold accordingly.

Question: Who are the key stakeholders you are working together with to leverage your solution?

Local partnerships. I can keep shouting this out until I am blue in the face. No one has any business riding into locations on a shiny white horse proposing solutions. Work with local organisations, governments, hospitals, in our case. We work with self-help women's groups, charities, the WHO, refugee accelerators.

Question: How does Hanai challenge the current healthcare system? Do you see a difference between Europe and the United States?

Healthcare systems are toxically conservative. It is time to push the boundaries and we need to continue to experiment how to do this. Hanai, together with its equal tech compatriots are here to look at novel ways but also look at how we can marry current usage of technology with healthcare needs in rural areas. The US, at least at its surface, seems more open to running with wild, new ideas and trying things out. In Europe, in Germany specifically, we tend to want tried and tested methods. Someone needs to try!

Question: What motivates you to disrupt healthcare in rural communities through blockchain technology and artificial intelligence? What are your aspirations / future plans?

I feel privileged to be in this sweet place where I create with technology. And I create solutions to save lives with technology, in my own small way. In a few years, I want my young boys to be able to say, my mother was among those who made the tech journey to ensure every child gets equal access to health.

\section{Conclusion}

It is evident that the current level of healthcare data is scattered and requires a new type of information infrastructure. Blockchain-based solutions offer different option for the short, medium and long term. Although each case study has a different focus and 
is in a different phase of development, they all offer insights into how to change (data) processes, information flows, and business models in healthcare to become more patient centred - thereby giving patients the power over their medical data and the ability to improve the healthcare industry.

The following part will highlight the main opportunities and challenges of remodelling the existing healthcare infrastructure based on the three case studies:

\section{Opportunities}

1. Increased data portability - all three solutions increase the interoperability of medical data. This is essential to make healthcare systems become more effective/efficient. Blockchain serves as a transaction layer that makes it possible to seamlessly share data with different stakeholders to gain insights and improve the health of a patient. Blockchain also serves as a link that integrates information from multiple sources such as mobile devices, wearableas and databases.

2. Patient data management - blockchain can improve the overall accessibility, transparency and security of the medical data. It puts the patient into the centre of the ecosystem, thereby ideally giving patients more power over their own health data.

3. Strengthen ecosystems - an increase in data portability can enable stakeholders to work together seamlessly. Improving efficiency can ultimately improve the lives of many patients.

\section{Challenges}

1. Siloes - the healthcare industry is heavily fragmented which makes is very hard to solve the interoperability challenge and share data seamlessly.

2. Standardization - it is tricky to standardize the information stored on a blockchain. Therefore precise standards are needed to strengthen the interoperability and exchange of medical data so that it adds value to all parties involved.

3. Partnerships - partnering with the right partners is essential to have an impact, they have to be open and able to work together very closely to find new ways to unlock the power of data driven healthcare. 